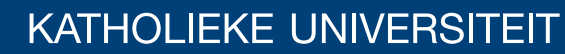 \\ LEUVEN
}

\section{Faculty of Business and Economics}

) ,; $\square[7 \mathrm{KH}) \mathrm{HDU}, \mathrm{QGH}$

P HDVXUQJIP DUWHMHDU

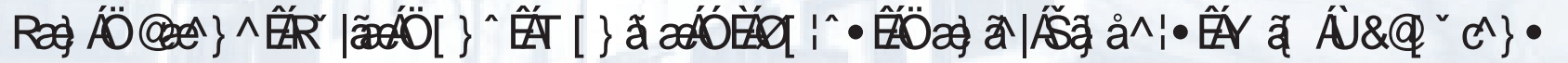

DEPARTMENT OF ACCOUNTANCY, FINANCE AND INSURANCE (AFI) 


\title{
FIX - The Fear Index measuring market fear
}

\author{
Jan Dhaene \\ K.U.Leuven, Leuven, Belgium \\ email: Jan.dhaene@econ.kuleuven.be \\ Monika B. Forys \\ K.U.Leuven, Leuven, Belgium \\ email: Monika.Forys@wis.kuleuven. be \\ Julia Dony \\ K.U.Leuven, Leuven, Belgium \\ email: julia.dony@student.kuleuven. be \\ Daniel Linders \\ K.U.Leuven, Leuven, Belgium \\ email: Daniel.Linders@econ.kuleuven.be \\ Wim Schoutens \\ K.U.Leuven, Leuven, Belgium \\ email: wim@schoutens.be
}

July 18, 2011

\section{Contents}

1 Introduction 2

2 Measuring market risk via the VIX 3

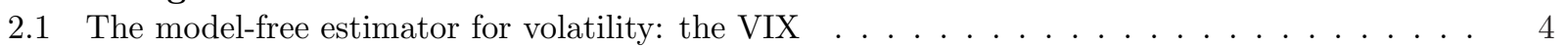

3 Measuring liquidity risk via the implied liquidity $\quad 6$

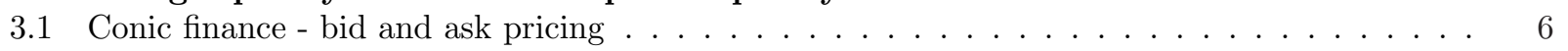

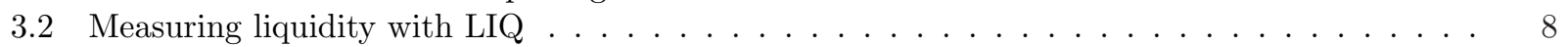

4 Measuring herd-behavior via the comonotonicity 9

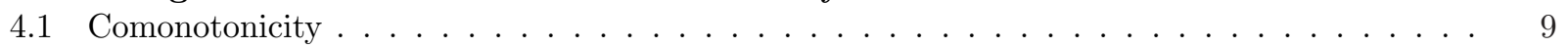

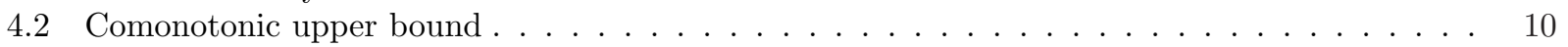

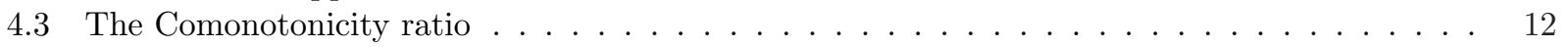

5 FIX: The Fear Index $\quad 14$

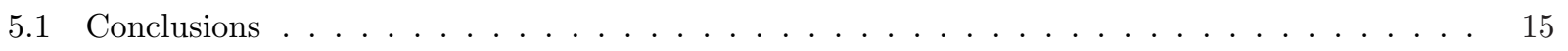

\begin{abstract}
In this paper, we propose a new fear index based on (equity) option surfaces of an index and its components. The quantification of the fear level will be solely based on option price data. The index takes into account market risk via the VIX volatility barometer, liquidity risk via the concept of implied liquidity, and systemic risk and herd-behavior via the concept of comonotonicity. It thus allows us to measure an overall level of fear (excluding credit risk) in the market as well as to identify precisely the individual importance of the distinct risk components (market, liquidity or systemic risk). As a side result we also derive an upperbound for the VIX.
\end{abstract}




\section{Introduction}

The VIX is a key measure of market expectations of near-term volatility conveyed by S\&P500 stock index option prices. It is often referred to as the fear index or fear gauge. Since its introduction in 1993, the VIX has been considered by many to be a good barometer of investor sentiment and market volatility. It is a weighted blend of prices for a range of options on the S\&P500 index. The formula uses a kernel-smoothed estimator that takes as inputs the current market prices for all out-of-the-money calls and puts for the front month and second month expirations. The goal is to estimate the implied volatility of the S\&P500 index over the next 30 days. On March 26, 2004, the first-ever trading in futures on the VIX Index was launched on the CBOE Futures Exchange (CFE). As of February 24, 2006, it became possible to trade VIX options contracts.

Actually, the VIX is an indicator of perceived volatility in either direction (including the upside) and hence not necessarily bearish for the stocks. Of course it is well documented that volatility and stock returns are negatively correlated.

Next to volatility, there are also other risk or fear factors in the market. Other fear components are for example systemic risk, liquidity risk and counterparty risk. More precisely, in times of heavy distress, besides very high levels of volatility, we typically observe also a drying up of liquidity in the sense that bid and ask spreads widen. When liquidity dries up, one cannot easily unwind positions near theoretical mid prices anymore, but one faces a negative price impact for immediate liquidiations; fire-sale transactions are typically at much lower prices. Furthermore, in such circumstances we also see more herd behavior pointing to a movement of the market into one direction. The later is related to the dependency relationships between traded assets. Finally, the market is well aware of the fact that in stress situations the probability that a counterparty fails is rising. Good indicators of counterpart risk are the credit indices like CDX and iTraxx.

In this paper, we will create a new fear index on the basis of (equity) option surfaces on an index and its components. The quantification of the fear level is hence on the basis of option price data only and not on any kind of historical data. The index will take into account market risk, via the VIX volatility barometer, liquidity risk, via the concept of implied liquidity, and finally systemic risk, via the concept of comonotonicity. The index allows us to measure an overall level of fear (excluding credit risk) in the market and to identify exactly the importance of the individual components (market, liquidity or systemic risk).

As indicated above the paper will make use of the concept of implied liquidity introduced in [13]. It is based on the fundamental theory of conic finance, in which the one-price theory is abandoned and replaced by a two-price theory giving bid and ask prices for traded assets. The pricing is performed by making use of non-linear distorted expectations. In essence, the distorted expectation used in [8] is parameterized by one parameter. A high value of this parameter gives rise to a wide bid-ask spread, a low value to a small spread. Given a market bid-ask spread, one can, via reverse engineering (cfr. implied volatility), back out the unique implied parameter to be put into the distortion function to recoup the market spread. This implied parameter is called the implied liquidity parameter. This allows us to measure the degree of liquidity of a certain asset in an isolated manner and to quantify it exactly.

Further, in order to quantify the level of systemic risk in the markets, we make use of the theory of comonotonicity (see [16] and [17]). This theory allows us to measure herd behavior, i.e. to which degree the whole market just goes into one direction. In particular, the comonotonic dependency structure is such that it is driven by one single systemic factor, and so that under a full comonotonic setting, all 
movements of all the assets are driven by this single factor. By pricing vanilla options on the index, which we see as options on a basket of the underlying components, under the comonotonic dependency structure and comparing these with actual index option prices, we are able to measure how far the observed market prices are away from the fully comonotonic market case. If we are in the theoretical case that the comonotonic gap, i.e. the difference between the comonotonic price and the market price, is zero, we are in a market driven purely by one common factor. If the gap is large, one is closer to a situation where the index components have a fully independent idiosyncratic behavior. The notion comonotonicity gap was introduces by Laurence ([21]). Comonotonic option prices can be determined via the general procedure presented in [16]. The applications of this procedure to the index option case in a market with a finite number of options traded is considered in [21] and [7].

This paper is organized as follows. First we elaborate on how exactly to compute the components of the overall market fear index. Then, we bring together the liquidity, the systemic and the volatility component into one overall fear estimate. We do this by taking a weighted sum, where the weights are set such that a number of fear of 100 represents historically the average case. A number above 100 indicates that the fear is above average; a number below 100 indicates that we are in a fear situation below average. The later is exactly quantified on the basis of a historical study over the period January 2007 - October 2009, for which we calculate the fear numbers on a daily basis for the Dow Jones Index and its components. Some key events in the recent credit crisis in that period are clearly identified.

\section{Measuring market risk via the VIX}

In 1993 the Chicago Board Options Exchange (CBOE) introduced a new index, called $\boldsymbol{V} \boldsymbol{I} \boldsymbol{X}$, which aimed at estimating the expected short-term volatility of the S\&P100 index over the next 30 days. Initially, VIX used to be an average of eight different implied volatilities calculated from eight at-the-money options of the S\&P100. In particular, two ATM calls and two ATM puts were selected for two different maturities (which we will refer to as "near term" and "next term" maturities) and the implied volatilities were computed according to a Black-Scholes model.

However, model dependent estimations based on the small range of options inaccurately reflected the real market volatilities. Thus, in September 2003 the new VIX has been introduced (see [6]). It is based on a much wider range of options and the underlying index has been replaced by the larger S\&P500, which provides stronger correlation with the market than S\&P100, as more stocks are involved. Also a model-free approach is used. This model-free approach relies on an volatility estimation developed in [4] combined with an efficient discretisation proposed in [20]. There is no model involved and the only requirements are continuity, absence of arbitrage and Markovian dynamics.

On March 26, 2004, the first-ever trading in futures on the VIX Index was launched on CBOE Futures Exchange (CFE). As of February 24, 2006, it became possible to trade VIX options contracts. On January 5, 2011, CBOE announced to also VIX-ify individual stocks like APPL, IBM, GS, GOOG, ....

The new VIX index is often referred to as the fear index or fear gauge, since its extreme values were achieved during the substantial decreases on the market. As mentioned, the volatility measure aims at estimating the expected short-term volatility of the S\&P500 index over the next 30 days. It is calculated using the current market prices of all out-of-the-money options with front month and second month expirations. Values of the VIX index based on S\&P500 are depicted in Figure 1.

Since its introduction, the VIX has been considered by many to be a good barometer of investor's sentiment and market volatility. The VIX typically spikes up when the market falls and goes down when 


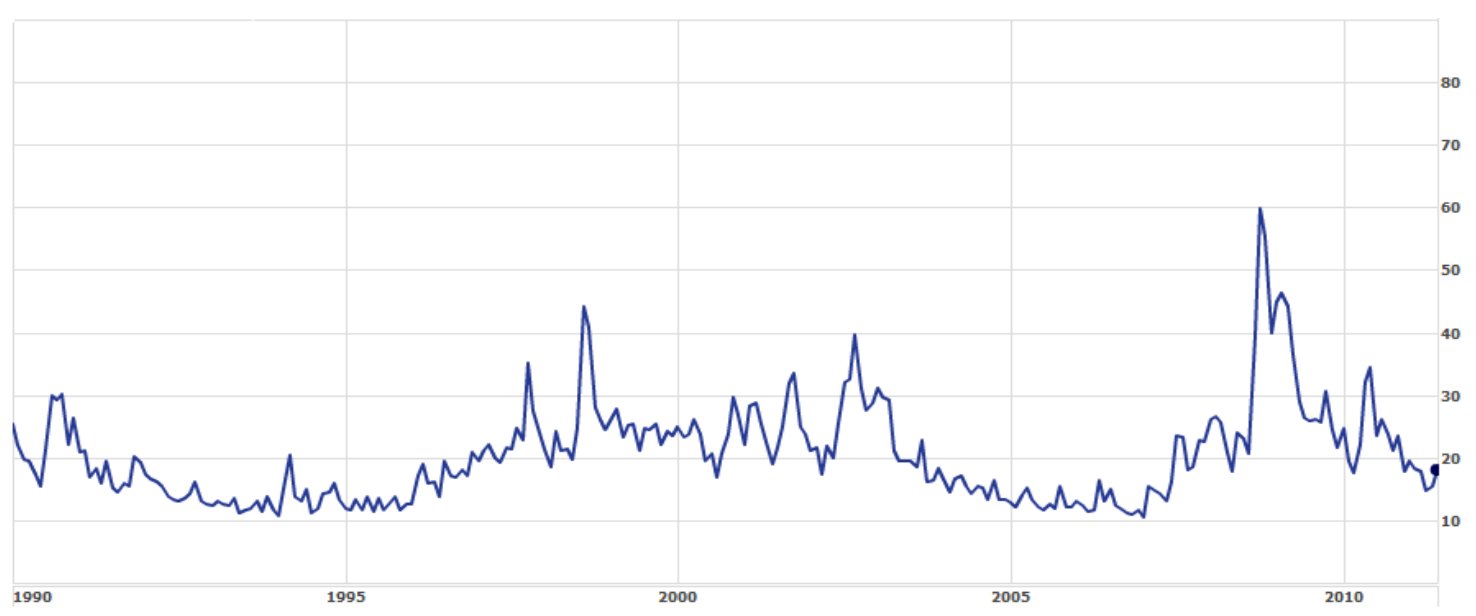

Figure 1: Historical values of the S\&P500 VIX; period 01.1990-12.2010

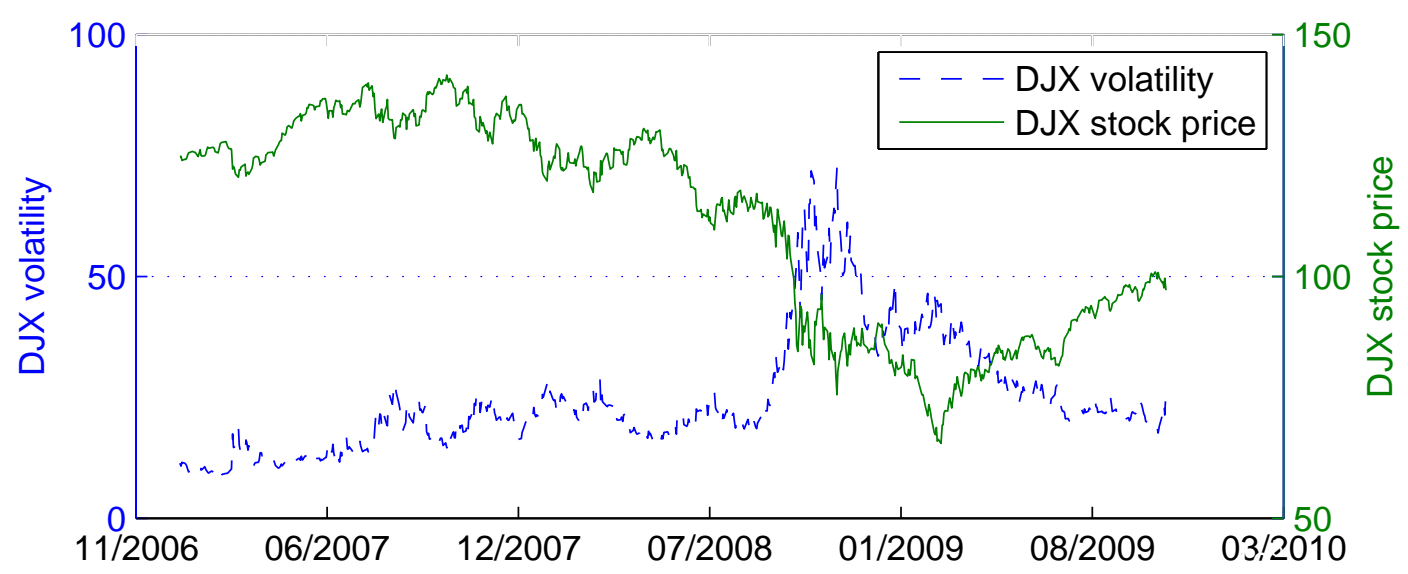

Figure 2: DJX volatility vs DJX stock price; period 01.2007-10.2009

the market goes up. This reflects a natural negative correlation between the VIX and the index returns (see Figure 2). The VIX thus quantifies the concept of volatility and acts as an effective measure for the expected movements in the next 30 days S\&P500 returns.

The VIX index typically fluctuated within a range of 15-30, with an average of 18.97 for the period 04.01.1993-31.12.2007. Due to the worldwide financial crisis in 2008, the VIX reached a value of 80 around November 2008 (see Figure 1).

The next subsection describes the notion of the model-free estimator for the volatility.

\subsection{The model-free estimator for volatility: the VIX}

As mentioned before, the VIX index has not always been calculated in the same way, as in September 2003 the model-free approach based on a wider option surface was introduced. The actual VIX is a weighted blend of prices for a range of options on the S\&P500 index. The formula uses as inputs the current market prices for all out-of-the-money calls and puts for the front month and second month expirations. The 
goal is to estimate the volatility of the S\&P500 index over the next 30 days.

The following quantity is crucial in the VIX calculation. It gives a model-free estimate for the variance, based only on options with maturity $T$.

$$
\sigma^{2}=\frac{2}{T} \sum_{i} \frac{\Delta K_{i}}{K_{i}^{2}} e^{r T} Q\left(K_{i}\right)-\frac{1}{T}\left(\frac{F}{K_{0}}-1\right)^{2}
$$

where

- $F$ is the forward index level. $F$ is determined by first identifying the strike price, $K^{*}$ at which the absolute difference between the call $\left(C\left(K^{*}, T\right)\right)$ and put $\left(P\left(K^{*}, T\right)\right)$ prices is the smallest. Then $F=K^{*}+e^{r T}\left(C\left(K^{*}, T\right)-P\left(K^{*}, T\right)\right)$;

- $K_{0}$ is the first strike below the forward index level;

- $K_{i}$ is the strike price of the $i$ th out-of -the money option; a call if $K_{i}>K_{0}$ and a put if $K_{i}<K_{0}$; both put and call if $K_{i}=K_{0}$. The range of the strikes taken into consideration is described in [10];

- $\Delta K_{i}$ is half the difference between the strikes on either side of $K_{i}$, i.e.

$$
\Delta K_{i}=\left(K_{i+1}-K_{i-1}\right) / 2
$$

except for the lowest strike, where $\Delta K$ is simply the difference between the lowest strike and the next higher strike. Likewise, $\Delta K$ for the highest strike is the difference between the highest strike and the next lower strike;

- $Q\left(K_{i}\right)$ is the midpoint of the bid/ask spread for each option with strike $K_{i}$; The $K_{0}$ put and call prices are averaged to produce a single value.

Here, $C(K, T)$ and $P(K, T)$ denote the respective mid-prices of the call and put options with strike $K$ and maturity $T$. In contrast, we write $C^{\text {bid }}(K, T)$ and $C^{\text {ask }}(K, T)$ for the bid and ask prices. One can notice that the VIX calculation is very much related to the implementation of a Variance Swap as elaborated on in [5], [24] and [15].

For the actual calculation of the VIX index, which is a 30-day forward looking estimate of the volatility, one needs to compute two variances based on this formula, namely a first one, $\sigma_{1}^{2}$, for the near term options $\left(T_{1}\right)$ and a second one, $\sigma_{2}^{2}$, for the next term options $\left(T_{2}\right)$. When the near-term options have less than a week to expiration, the VIX "rolls" to the second and third contract months. The VIX is then an interpolation at the 30 days point, based on values at $T_{1}$ and $T_{2}$ :

$$
V I X_{30}=100 \sqrt{\{T_{1} \sigma_{1}^{2} \underbrace{\left[\frac{N_{T_{2}}-N_{30}}{N_{T_{2}}-N_{T_{1}}}\right]}_{x_{1}}+T_{2} \sigma_{2}^{2} \underbrace{\left[\frac{N_{30}-N_{T_{1}}}{N_{T_{2}}-N_{T_{1}}}\right]}_{1-x_{1}}\} \frac{N_{365}}{N_{30}}}
$$

where:

- $N_{T_{1}}=$ number of minutes to settlement of the near-term options (i.e. with maturity $T_{1}$ );

- $N_{T_{2}}=$ number of minutes to settlement of the next-term options (i.e. with maturity $T_{2}$ );

- $N_{30}=$ number of minutes in 30 days;

- $N_{365}=$ number of minutes in a 365-day year. 


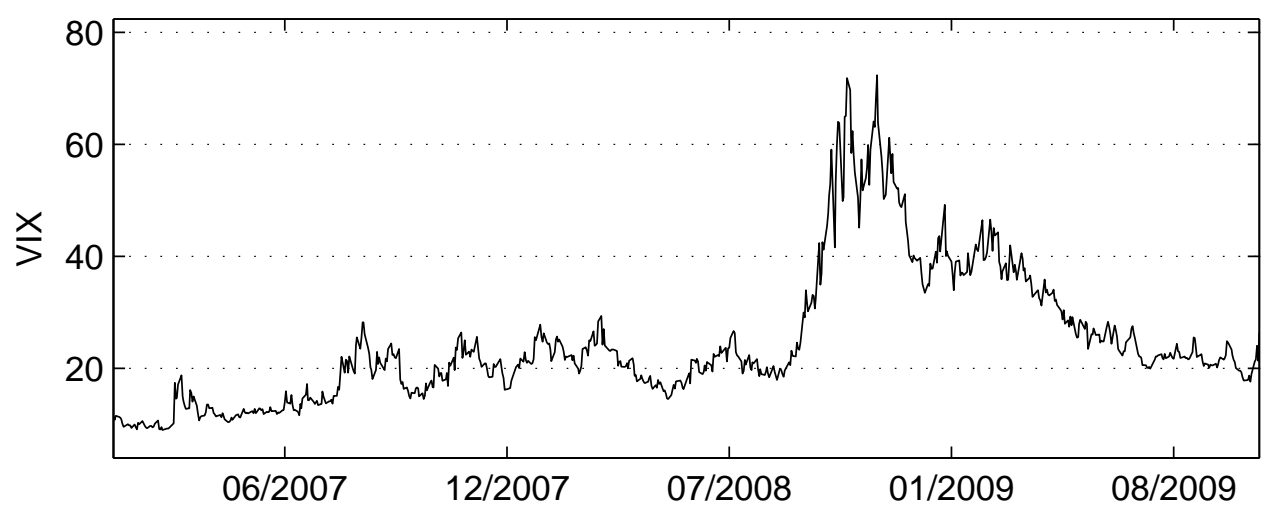

Figure 3: Dow Jones VIX; period 01.2007-10.2009

The VIX methodology was historically introduced on the S\&P500 options and later applied to several other indices, stocks and assets. The VIX calculations conducted in this research will in contrast be based on the Dow Jones Industrial Average Index (DJX). Figure 3 shows the DJX VIX estimate in the period between January 2007 and October 2009.

One can observe a mean-reverting behavior of the VIX. In the period preceding the credit crisis, the VIX underwent a rapid growth and went from a value of 20 up to a value of more than 70 in a timespan of a few weeks only. The S\&P500 VIX actually went up even to 80. Hence at that point, at the heat of the financial crisis, the market was expecting unusually large movements of the stocks. We also remark that in 2010, the DJX VIX has come down from its highest levels back under the 30 level again. The average Dow Jones VIX level for this period is calculated as 24.66.

\section{Measuring liquidity risk via the implied liquidity}

In the previous section it was shown that volatility levels can give us an indication of the nervousness of the market conditions. Liquidity is another important measure, which reflects an asset's ability to be sold. High bid-ask spreads characterize illiquid products, whereas liquidity implicates a smaller spread. However, it is very difficult to measure liquidity in an isolate manner. Bid-ask spreads can move around in a non-linear manner if spot or volatility moves, without a change in liquidity.

In the sequel, we will discuss the concept of implied liquidity, which in a unique and fundamental founded way isolates and quantifies the liquidity risk in financial markets. This concept was already proposed in [13] and is based on the theory of conic finance, in which the one-price theory is abandoned and the two-price market is employed.

\subsection{Conic finance - bid and ask pricing}

In this section, we summarize the basic conic finance techniques needed to calculate the implied liquidity parameter related to a vanilla option position. For more background, see [8], [9] and [23]. Conic finance uses distortion functions to calculate distorted expectations. In [13], a distortion function from the minmaxvar family parameterized by a single parameter $\lambda \geq 0$ as in Equation (3) is chosen. 


$$
\Phi(u ; \lambda)=1-\left(1-u^{\frac{1}{1+\lambda}}\right)^{1+\lambda}
$$

Hereafter, we will employ distorted expectations to calculate bid and ask prices. The prices arise from the theory of acceptability. A risk $X$ is said to be acceptable (notation: $X \in \mathcal{A}$ ) if

$$
E_{Q}[X] \geq 0 \text { for all measures } Q \text { in a convex set } \mathcal{M} \text {. }
$$

The convex set $\mathcal{M}$ contains the supporting measures, which can be seen as a kind of test-measures under which the cash-flow $X$ needs to have positive expectation to deliver acceptability. Under a larger set $\mathcal{M}$, one has a smaller set of acceptable risks, because there are more underlying tests to be passed.

Operational cones were defined by Cherney and Madan [8] and depend solely on the distribution function $G(x)$ of $X$ and a distortion function $\Phi$. Here $X \in \mathcal{A}$ if the distorted expectation is non-negative. More precisely, the distorted expectation with respect to the distortion function $\Phi$ (we use the one given in Equation (3) but other distortion functions are also possible), of a random variable $X$ with distribution function $G(x)$, is defined as

$$
\operatorname{de}(X ; \lambda)=\int_{-\infty}^{+\infty} x \mathrm{~d} \Phi(G(x) ; \lambda) .
$$

Note that if $\lambda=0, \Phi(u ; 0)=u$ and hence $d e(X ; 0)=\mathrm{E}[X]$ is equal to the original expectation.

The ask price of payoff $X$ is determined by

$$
\operatorname{ask}(X)=-\exp (-r T) \operatorname{de}(-X ; \lambda)
$$

This formula is derived by noting that the cash-flow of selling $X$ at its ask price is acceptable in the relevant market, that is $\operatorname{ask}(X)-X \in \mathcal{A}$. Similarly, the bid price of payoff $X$ is determined as

$$
\operatorname{bid}(X)=\exp (-r T) \operatorname{de}(X ; \lambda)
$$

Here the cash-flow of buying $X$ at its bid price is acceptable in the relevant market : $X-b i d(X) \in \mathcal{A}$.

One can prove that the bid and ask prices of a positive contingent claim $X$ with distribution function $G(x)$ can be calculated as

$$
\begin{aligned}
& \operatorname{bid}(X)=\exp (-r T) \int_{0}^{+\infty} x \mathrm{~d} \Phi(G(x) ; \lambda), \\
& \operatorname{ask}(X)=\exp (-r T) \int_{-\infty}^{0}(-x) \mathrm{d} \Phi(1-G(-x) ; \lambda) .
\end{aligned}
$$

Suppose now that we are given a market bid and ask price for a European call. We can then calculate the mid price of that call option, as the average of the bid and ask prices. Out of this mid price we calculate the implied Black-Scholes volatility, to calculate the conic bid and ask prices (using the implied volatility as parameter). Under the Black-Scholes framework, this comes down to the following calculations for an European call option with strike $K$ and maturity $T$. The distribution of the call payoff random variable to be used in (5) and (6) is in this case given by

$$
G(x)=1-\mathrm{N}\left(\frac{\log \left(S_{0} /(K+x)\right)+\left(r-q-\sigma^{2} / 2\right) T}{\sigma \sqrt{T}}\right), \quad x \geq 0
$$

where $S_{0}$ is the current stock price, $r$ the risk-free rate and $q$ the dividend yield. Further, $\mathrm{N}$ is the cumulative distribution function of the standard normal law and $\sigma$ is the implied vol determined on the basis of the mid price. For $x<0, G(x)=0$, since the payoff is a positive random variable. The above closed-form solution for $G(x)$ in combination with Equation (5) and (6) gives rise to very fast and accurate calculations of the bid and ask prices. 


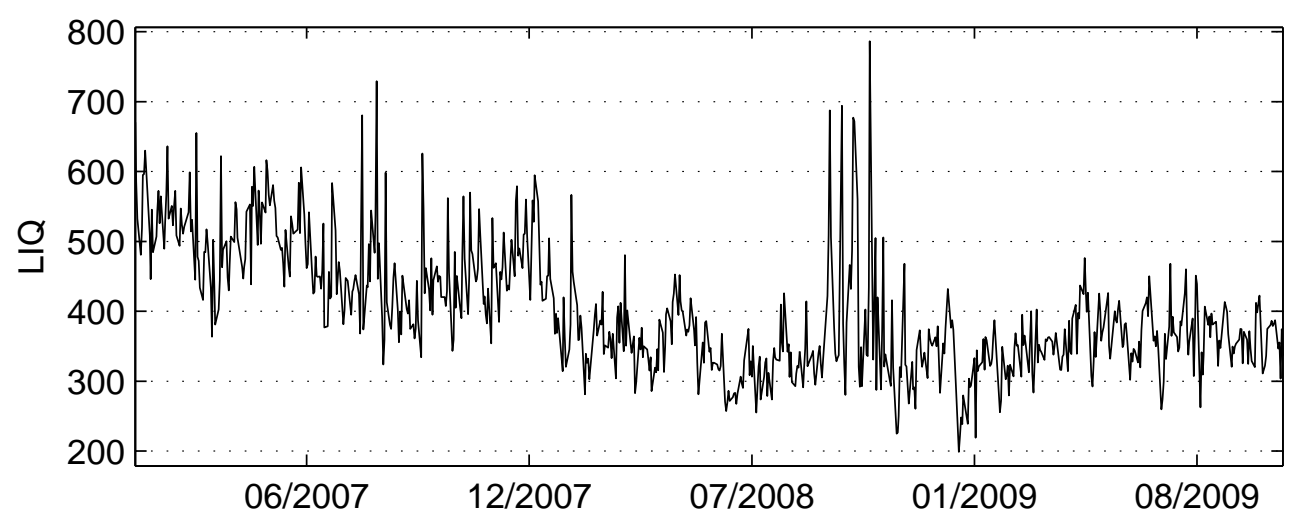

Figure 4: Dow Jones LIQ; period 01.2007-10.2009

The parameter $\lambda$ in (5) and (6), which fits the bid-ask spread around the mid price the best, is then called the implied liquidity parameter. The smaller the implied liquidity parameter, the more liquid the underlying and the smaller the bid-ask spread. In the extremal case where the implied liquidity parameter equals 0 , the bid price coincides with the ask price, and we are back in the one-price framework.

\subsection{Measuring liquidity with LIQ}

It is well-known that a distressed market suffers from drying liquidity. In order to measure the liquidity risk, we propose a measure based on implied liquidity, which we will call LIQ.

We denote by $L I Q_{j}$ the 30-days implied liquidity of company $j$, calculated from the near and next term implied liquidities: $\lambda_{j}^{*}\left(T_{1}\right)$ and $\lambda_{j}^{*}\left(T_{2}\right)$. We compute it using the same weights as in the VIX methodology. $\lambda_{j}^{*}\left(T_{i}\right), i=1,2$ itself is calculated as an average of all the individual implied liquidities of all non-zero bid call and put options of company $j$. Hence, $L I Q_{j}$ of the $j$-th company is given by

$$
L I Q_{j}=x_{1} \lambda_{j}^{*}\left(T_{1}\right)+\left(1-x_{1}\right) \lambda_{j}^{*}\left(T_{2}\right)
$$

In the same way we calculate the implied liquidity $L I Q_{D J X}$ of the index. This combination of near and next term liquidities provides a short term forward looking implied liquidity.

The overall liquidity index for a particular day is defined as:

$$
\mathrm{LIQ}=\frac{1}{2} \mathrm{LIQ}_{D J X}+\frac{1}{2 n} \sum_{j=1}^{n} \mathrm{LIQ}_{j} .
$$

In Figure 4 the market liquidity estimation based on the DJX index and all the 30 underlying stocks is presented. We clearly observe that LIQ is not constant over time and apparently exhibits a mean-reverting behavior. Recent work investigates this stochastic liquidity behavior more in depth, see [2].

The long run average of the implied liquidity of the data set in the period between January 2007 and October 2009 equals $412 \mathrm{bp}$. The highest value of the LIQ parameter, $1260 \mathrm{bp}$, was reached on the 24th of October 2008. Around this day several European banks were rescued by government intervention. 


\section{Measuring herd-behavior via the comonotonicity}

In this section, we introduce a third ingredient contributing to the general panic level in the market, namely herd behavior. This notion refers to the tendency of one decision maker to take his decisions in accordance with those of a whole group of decision makers, whether or not these decisions are rational.

When the market is more agitated, it is therefore not unusual to observe a stronger herd behavior pointing to a systemic movement of the market into one direction. Measuring the risk of herd behavior is not necessarily straightforward, as it relies on human's reactions in specific circumstances, which are not easy to quantify. However, the herd phenomenon in the financial market is also essentially related to the dependency relationship between the traded assets.

Inspired by [18], we propose a measure for herd behavior in the market based on the concept of comonotonicity. While in [18], the implied variance of the index price is compared with its comonotonic version and its ratio is called the HIX, we propose to compare the VIX index by its comonotonic version. From a methodological point of view this is very similar to the HIX approach. Therefore, for a more profound study of the underlying methodology, we refer to [18]. For an overview of the theory of comonotonicity and its applications in insurance and finance, we refer to [16] and [17]. For further work on the applications of this theory in an option pricing framework, we refer to [7], [1], [25] and [22]. A recent overview of the literature on financial applications of the theory of comonotonicity is given in [14].

\subsection{Comonotonicity}

In this section, we will summarize basic concepts of comonotonicity theory in relationship with the dependency structure between the underlyings in a basket of assets. We start with stating some main results concerning comonotonicity theory. Definitions, results and detailed proofs can be found in [16] and [7]. Let us consider $n$ different stocks $i=1, \ldots, n$ with corresponding stock price processes $\left\{S_{i}(t), t \geq 0\right\}$. These stocks form an index (or basket) consisting of a combination of a certain amount $w_{i}$ of stock $i$, where $w_{1}, w_{2}, \ldots, w_{n}$ is a series of upfront fixed positive weights. We denote by $\{\mathcal{S}(t), t \geq 0\}$ the price process of the index calculated as the weighted sum of the $n$ underlying stock price components, i.e.,

$$
\mathcal{S}(t)=\sum_{i=1}^{n} w_{i} S_{i}(t), \quad t \geq 0 .
$$

In our example, we will use $n=30$, and work with the 30 components $\left(S_{i}, i=1, \ldots, 30\right)$ of the DJX index $(\mathcal{S})$.

Suppose there exists an option market of vanilla calls and puts on the individual stocks $i=1, \ldots, n$, as well as on the index. We shall denote by $C_{i}(K, T)$ and $P_{i}(K, T)$ the prices of a European call option and European put option resp. on stock $i$ with strike $K$ and maturity $T$. In the same way, we write $\mathcal{C}(K, T)$ and $\mathcal{P}(K, T)$ for the option prices on the index.

Recall that the payoff of an European call with strike $K$ and maturity $T$ on the index is given by $(\mathcal{S}(T)-K)^{+}=\left(\sum_{i=1}^{n} w_{i} S_{i}(T)-K\right)^{+}$. In order to compute the price of this call option, one would actually need to have full knowledge about the dependency structure of the underlying stocks. This information is usually not known, however, one can always find an optimal upper bound of $\mathcal{C}(K, T)$ by taking a linear combination of observable call option prices $C_{i}(K, T)$, and which corresponds to the case when the stock price vector is comonotonic. This leads us to the definition of comonotonic vectors.

Definition 1 (Comonotonic vector) Let $Y_{1}, \ldots, Y_{n}$ be arbitrary random variables and let $U$ be a uniformly distributed random variable on the unit interval. We say that the random vector $\mathbf{Y}=\left(Y_{1}, \ldots, Y_{n}\right)$ 
is comonotonic if

$$
\mathbf{Y} \stackrel{d}{=}\left(F_{Y_{1}}^{[-1]}(U), \ldots, F_{Y_{n}}^{[-1]}(U)\right),
$$

where $\stackrel{d}{=}$ stands for equal in distribution, and $F_{Y}^{[-1]}(u)=\inf \left\{x \in \mathbb{R}: F_{Y}(x) \geq u\right\}$. (and $\inf \emptyset=+\infty$ by convention).

The comonotonic vector is driven by only one single factor $(U)$ - the systemic risk. Now, for any random vector $\mathbf{X}=\left(X_{1}, \ldots, X_{n}\right)$, we can define the so-called comonotonic counterpart of $\mathbf{X}$. It is denoted by $\mathbf{X}^{c}$ and is defined as

$$
\mathbf{X}^{c} \equiv\left(X_{1}^{c}, \ldots, X_{n}^{c}\right) \stackrel{d}{=}\left(F_{X_{1}}^{[-1]}(U), \ldots, F_{X_{n}}^{[-1]}(U)\right)
$$

In this context, we define the comonotonic index price process as:

$$
\mathcal{S}^{c}(t)=\sum_{i=1}^{n} w_{i} S_{i}^{c}(t), \quad t \leq T
$$

where $S^{c}(t)=\left(S_{1}^{c}(t), \ldots, S_{n}^{c}(t)\right)$ is the comonotonic counterpart of the stock price vector $S(t)=\left(S_{1}(t), \ldots, S_{n}(t)\right)$. In analogy to the regular index call price we will denote

$$
\mathcal{C}^{c}(K, T)=e^{-r T} \mathbb{E}_{Q}\left[\left(\mathcal{S}^{c}(T)-K\right)^{+}\right] .
$$

for the comonotonic call value. Note that the comonotonic version incorporates perfect herd behavior, and index call options under perfect herd behavior should intuitively be more expensive, since each index component moves in the same direction and hence the index exhibits a higher volatility. From now on, to avoid unnecessary overloading of notation, we will omit writing " $(t)$ " whenever there is no confusion possible. In particular, we will write $\mathcal{S}^{c} \equiv \mathcal{S}^{c}(T)$ and $S_{i}(T) \equiv S_{i}$.

\subsection{Comonotonic upper bound}

In this section it will be shown how to derive an upper bound for a call option on the index in terms of call options on the individual stocks. For details, we refer to [7].

In fact comonotonicity theory implies, that it is always possible to bound the index option price $\mathcal{C}(K, T)$ from above, namely with the price of the comonotonic index call price $\mathcal{C}^{c}(K, T)$. To do so, we first have to specify the comonotonic distribution $F_{\mathcal{S}^{c}}$.

Theorem 2 (Comonotonic distribution) The distribution function of the comonotonic index price process is given for any $x \in\left(F_{\mathcal{S}^{c}}^{-1+}(0), F_{\mathcal{S}^{c}}^{-1}(1)\right)$ as

$$
F_{\mathcal{S}^{c}}(x)=\sup \left\{p \in[0,1]: \sum_{i=1}^{n} w_{i} F_{S_{i}}^{[-1]}(p) \leq x\right\} .
$$

where for each $0<\alpha \leq 1$ the alpha-inverse of $F_{\mathcal{S}^{c}}$ is given by

$$
F_{\mathcal{S}^{c}}^{[-1(\alpha)]}(p)=\sum_{i=1}^{n} w_{i} F_{S_{i}}^{[-1(\alpha)]}(p), \quad 0<\alpha \leq 1,
$$

and the alpha-inverse is defined as $F_{Y}^{[-1(\alpha)]}(u)=\alpha F_{Y}^{[-1]}(u)+(1-\alpha) F_{Y}^{[-1+]}(u), 0<\alpha<1$, with $F_{Y}^{[-1+]}(u)=\sup \left\{x \in \mathbb{R}: F_{Y}(x) \leq u\right\}$ (with $\sup \emptyset=-\infty$, by convention).

We are now able to calculate the expected payoff of a call option on the comonotonic basket, as it is shown in the following theorem; for a proof see [7]. 
Theorem 3 (Comonotonic index option price) Let $\mathcal{S}^{c}$ be the comontonic price process of an index as above. Then

$$
\mathbb{E}\left[\left(\mathcal{S}^{c}-K\right)^{+}\right]=\sum_{i=1}^{n} w_{i} \mathbb{E}\left(S_{i}-F_{S_{i}}^{[-1(\alpha)]}\left(F_{\mathcal{S}^{c}}(K)\right)\right)^{+},
$$

where $\alpha \in[0,1]$ must be chosen in such a way that $F_{\mathcal{S}^{c}}^{[-1(\alpha)]}\left(F_{\mathcal{S}^{c}}(K)\right)=K$, or equivalently (by the additivity property of comonotonic quantiles),

$$
\sum_{i=1}^{n} w_{i} F_{S_{i}}^{[-1(\alpha)]}\left(F_{\mathcal{S}^{c}}(K)\right)=K
$$

Consequently, the comonotonic index option price is given by

$$
\mathcal{C}^{c}(K, T)=\sum_{i=1}^{n} w_{i} C_{i}\left(F_{S_{i}}^{[-1(\alpha)]}\left(F_{\mathcal{S}^{c}}(K)\right), T\right) .
$$

Essentially, the above expression tells us that the price of a call option with strike $K$ and maturity $T$ on the index under the comonotonic setting equals a weighted sum of call prices on the index components. The weights are identical to the ones used for the index composition and the maturities are identical as well. The strikes are given by

$$
K_{i}^{*}=F_{S_{i}}^{[-1(\alpha)]}\left(F_{\mathcal{S}^{c}}(K)\right) .
$$

To determine these strikes we need to know the distribution functions of $S_{i}, F_{S_{i}}(x)$ and the distribution function of the comonotonic index $F_{\mathcal{S}^{c}}$. The distribution function of $F_{S_{i}}(x)$ can be extracted from the option surface of stock $i$ :

$$
F_{S_{i}}(x)=1+\left.e^{r T} \frac{\partial C(K, T)}{\partial K}\right|_{K=x+}, \quad x>0 .
$$

Given the marginal distribution functions, the comonotonic distribution function can be calculated using (8). Note that both (8) and (11) can be calculated in a model free way using only option price data.

The above formula (11) is however only valid if call options are available for any strike. In the real world this is not the case and only a finite number of call prices are available for a given maturity. Therefore, in [19] (see also [7]) one proposes to approximate $F_{S_{i}}(x)$ by a piecewise constant function $\bar{F}_{S_{i}}(x)$ defined as

$$
\bar{F}_{S_{i}}\left(K_{i, j}\right)=1+e^{r T} \frac{C_{i}\left(K_{i, j+1}, T\right)-C_{i}\left(K_{i, j}, T\right)}{K_{i, j+1}-K_{i, j}},
$$

where $K_{i, j}, j=1, \ldots, m_{i}$, are the traded strikes for the underlying stock $i$. Finally, we have that

$$
\bar{F}_{\mathcal{S}^{c}}(x)=\sup \left\{p \in[0,1]: \sum_{i=1}^{n} w_{i} \bar{F}_{S_{i}}^{[-1]}(p) \leq x\right\} .
$$

Having all the formulas at hand, we can define the comonotonic upper bound: for all strikes $K$ in the support of $F_{\mathcal{S}^{c}}$ we can bound the index option price by

$$
\mathcal{C}(K, T) \leq \sum_{i \in \mathcal{N}_{K}} w_{i} C_{i}\left(K_{i, j_{i}}, T\right)+\sum_{i \notin \mathcal{N}_{K}} w_{i}\left\{\alpha_{K} C_{i}\left(K_{i, j_{i}}, T\right)+\left(1-\alpha_{K}\right) C_{i}\left(K_{i, j_{i}+1}, T\right)\right\},
$$

where $j_{1}, \ldots, j_{n}$ and $\mathcal{N}_{K}$ are (sets of) indices depending on $F_{\overline{\mathcal{S}}^{c}}(K)$, and where $\alpha_{K}$ is a function of observed call option prices $C_{i}\left(K_{i, j}, T\right)$ only. In particular, we have that

- 1. the indices $j_{i}$ are such that $\bar{F}_{S_{i}}\left(K_{i, j_{i}-1}\right)<F_{\overline{\mathcal{S}}^{c}}(K) \leq \bar{F}_{S_{i}}\left(K_{i, j_{i}}\right)$,

2. $\mathcal{N}_{K}=\left\{i \leq n: F_{\overline{\mathcal{S}}^{c}}(K) \neq \bar{F}_{S_{i}}\left(K_{i, j_{i}}\right)\right\}$, 
3. $\alpha_{K}$ is any number satisfying $\bar{F}_{\mathcal{S}^{c}}^{\left[-1\left(\alpha_{K}\right)\right]}\left(F_{\overline{\mathcal{S}}^{c}}(K)\right)=K$, or equivalently,

$$
\sum_{i=1}^{n} w_{i} \bar{F}_{S_{i}}^{\left[-1\left(\alpha_{K}\right)\right]}\left(F_{\overline{\mathcal{S}}^{c}}(K)\right)=K .
$$

The comonotonic upper bounds can be computed for and on the basis of put options as well [22]. There is only one formula that requires adaptation, i.e. the empirical distribution function $\bar{F}_{S_{i}}\left(K_{i, j}\right)$. The expression in (12) then becomes

$$
\bar{F}_{S_{i}}\left(K_{i, j}\right)=e^{r T} \frac{P_{i}\left(K_{i, j+1}, T\right)-P_{i}\left(K_{i, j}, T\right)}{K_{i, j+1}-K_{i, j}} .
$$

The comonotonic upper bound for the index put option is then the analogue of (13), and can be formulated in the following way: For all strikes $K$ in the support of $F_{\overline{\mathcal{S}}^{c}}$ we can bound the index put option price by

$$
\mathcal{P}(K, T) \leq \sum_{i \in \mathcal{N}_{K}} w_{i} P_{i}\left(K_{i, j_{i}}, T\right)+\sum_{i \notin \mathcal{N}_{K}} w_{i}\left\{\alpha_{K} P_{i}\left(K_{i, j_{i}}, T\right)+\left(1-\alpha_{K}\right) P_{i}\left(K_{i, j_{i}+1}, T\right)\right\}
$$

where $j_{1}, \ldots, j_{n}$ and $\mathcal{N}_{K}$ are (sets of) indices depending on $F_{\overline{\mathcal{S}}^{c}}(K)$, and where $\alpha_{K}$ is a function of observed put option prices $P_{i}\left(K_{i, j}, T\right)$ only.

\subsection{The Comonotonicity ratio}

Knowing both the index option price $\mathcal{C}(K, T)$ for a certain strike $K$ and maturity $T$ and its upper bound $\sum_{i=1}^{n} w_{i} C_{i}\left(K_{i}^{*}, T\right)=\mathcal{C}^{c}(K, T)$, one can compare both values to measure how far one is away from the fully comonotonic situation. On the basis of this, [21] proposes the so-called comonotonicity gap, which compares the market's price with the perfectly comonotonic price by means of their ratio. We work with the related comonotonicity ratio:

$$
\varrho_{\text {call }}(K, T)=\frac{\mathcal{C}(K, T)}{\mathcal{C}^{c}(K, T)} .
$$

Alternatively, in order to have a more robust and overall comonotonicity measure and based on similar ideas as the one proposed in [18], we VIX-ify the above comonotonicity ratio by replacing call and put option quotes in the VIX formula by their comonotonic upper bound. More precisely, in (1) we replace $Q\left(K_{i}\right)$ by its comonotonic upper bound $Q^{c}\left(K_{i}\right)$, calculated according to the formulas above. This results in the following formula:

$$
\sigma_{\text {com }}^{2}(T)=\frac{2}{T} \sum_{i} \frac{\Delta K_{i}}{K_{i}^{2}} e^{r T} Q^{c}\left(K_{i}\right)-\frac{1}{T}\left(\frac{F}{K_{0}}-1\right)^{2} .
$$

In this way and using the same interpolation on the 30-days point (using the front and next month maturities), we derived a comonotonic $V I X\left(V I X^{c}\right)$, which is a market implied upper bound for the VIX (Figure 5):

$$
V I X^{c}=100 \sqrt{\{T_{1} \sigma_{\text {com }}^{2}\left(T_{1}\right) \underbrace{\left[\frac{N_{T_{2}}-N_{30}}{N_{T_{2}}-N_{T_{1}}}\right]}_{x_{1}}+T_{2} \sigma_{c o m}^{2}\left(T_{2}\right) \underbrace{\left[\frac{N_{30}-N_{T_{1}}}{N_{T_{2}}-N_{T_{1}}}\right]}_{1-x_{1}}\} \frac{N_{365}}{N_{30}}}
$$

We graph in Figure 5, the DJX VIX and its upperbound $V I X^{c}$. 


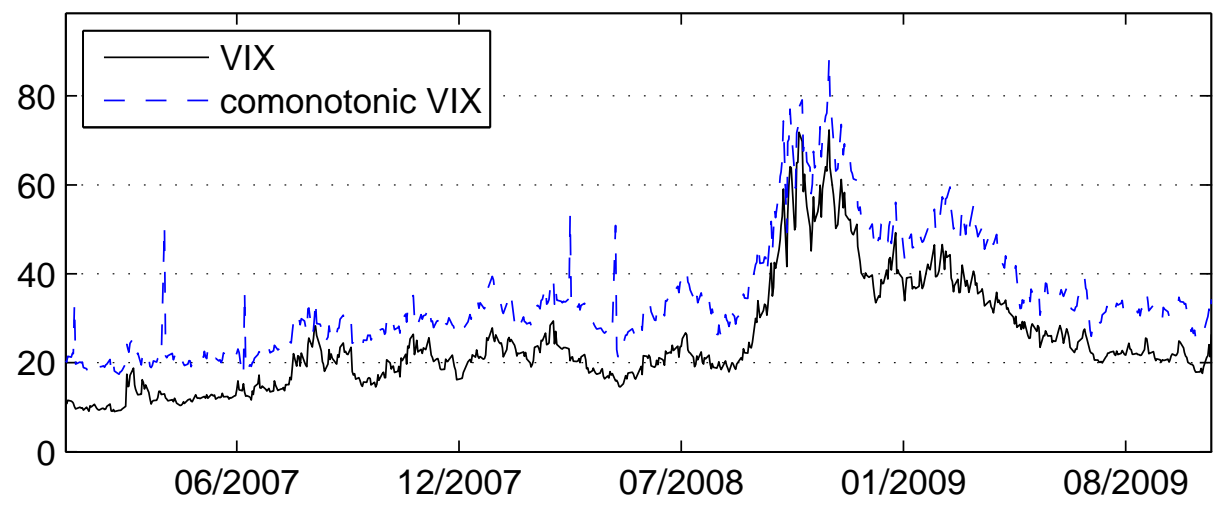

Figure 5: Dow Jones VIX and VIX ${ }^{c}$; period 01.2007-10.2009

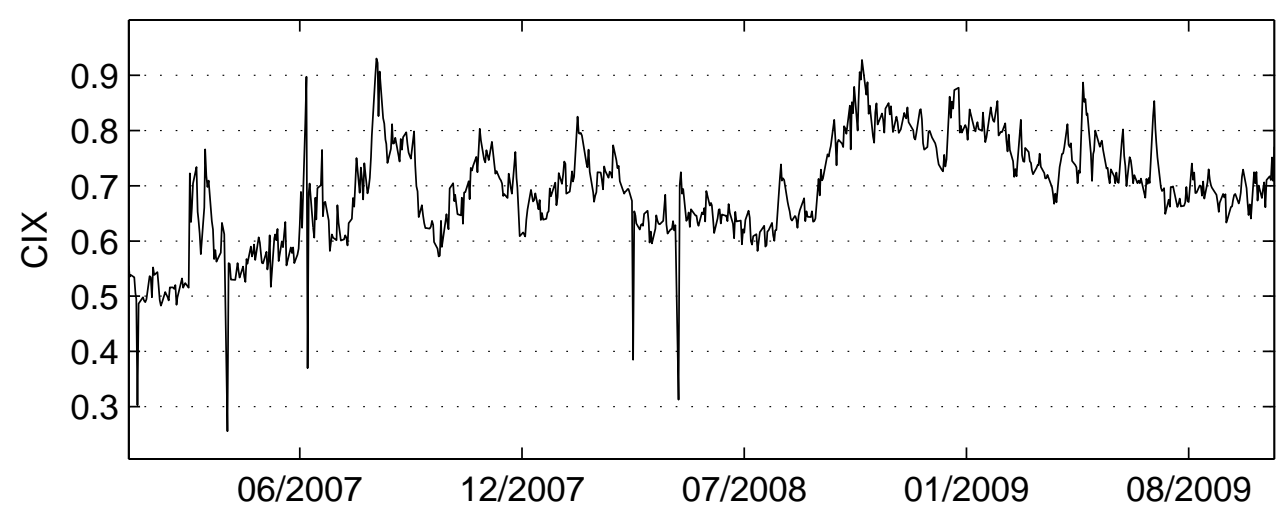

Figure 6: Dow Jones CIX; period 01.2007-10.2009

Finally, we define the comonotonicity VIX ratio, baptized CIX, as the ratio of the regular VIX and the comonotonic VIX, i.e.,

$$
C I X=\varrho_{V I X}=\frac{V I X}{V I X^{c}} .
$$

The CIX can be used as a measure for systemic risk and herd behavior. The closer to 1, the closer we are to the comonotonic situation and the more systemic risk or herd behavior there is in the market. Perfect herd behavior is reached when $C I X=1$. Hence, the ratio gives us a simple and convenient way to measure how much herd behavior is present in the market, and thus to quantify the systemic risk on the basis of traded option information.

Again, the credit crisis is clearly visible around October 2008 as well as financial issues during the summer of 2007. 


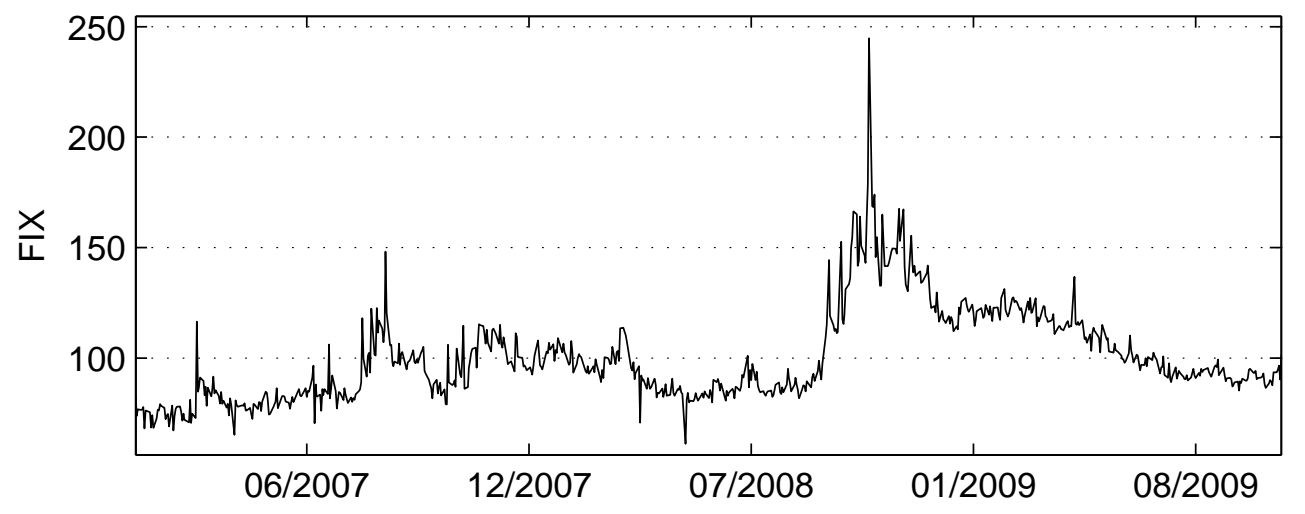

Figure 7: Dow Jones Fear Index; period 01.2007-10.2009

\section{FIX: The Fear Index}

In the previous chapters we have proposed measures for quantifying several types of risks in the market. As such, we have discussed the VIX as a (model-free) estimate for market risk, introduced LIQ as (model dependent) estimate for the liquidity and elaborated on the CIX as a (model-free) measure of herd behavior and systemic risk. The major objective of these developments was the establishment of a general measure for overall market fear, which is based on the three aforementioned components, combined in a particular way.

We call our new fear index the FIX. And, FIX is calculated out of VIX, LIQ and CIX as follows:

$$
F I X=\omega_{1} V I X+\omega_{2} L I Q+\omega_{3} C I X
$$

where $\omega_{1}, \omega_{2}, \omega_{3}$ are the weights allocated to the different risk measures in such a way that the contribution of each risk is proportional to its contribution to the "average fear situation". Based on our previous results and calculations, the respective average values for the DJX Index over the period $2007-2009$ are estimated as follows:

$$
\widetilde{\mathrm{VIX}}=24.66 \%, \widetilde{\mathrm{LIQ}}=400.65 \mathrm{bp} \text { and } \widetilde{\mathrm{CIX}}=69.16 \%
$$

We now define the weights $\omega_{1}, \omega_{2}$ and $\omega_{3}$ in such a way that

$$
0.25 \omega_{1}=0.04 \omega_{2}=0.7 \omega_{3}=\frac{100}{3}
$$

where the choices 25\%,400bp and $70 \%$ are settled in accordance with the obtained averages. Applying these values leads to the contribution of each component in the FIX:

$$
\omega_{1}=133.33, \quad \omega_{2}=833.33 \quad \omega_{3}=47.62 .
$$

These choices will then lead to a fear measure FIX having an average level of 100. A value FIX > 100 will reflect a market with a fear level above average, whereas a value FIX $<100$ expresses less fear in the market than average. Application of these values in the calculation of the FIX gives the following plot of the Fear Index FIX as shown in Figure 7. 


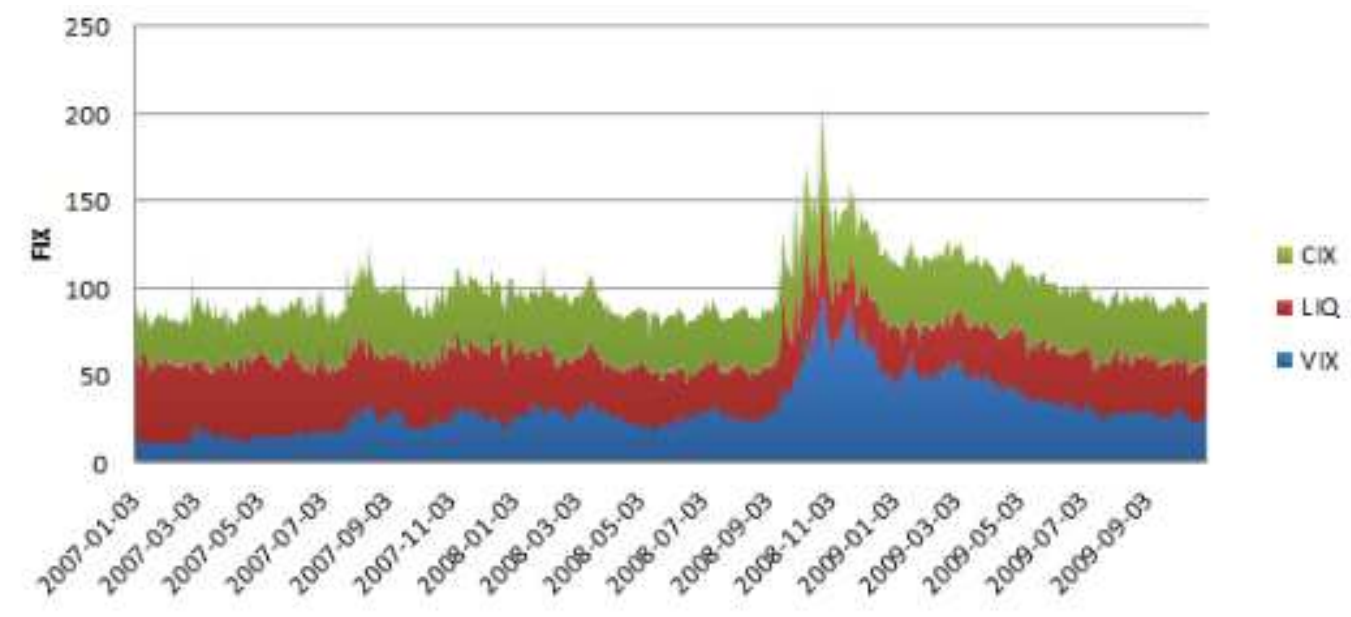

Figure 8: Dow Jones Fear Index components; period 01.2007-10.2009

The different market fear components are shown in Figure 8. The pattern of Figure 8 clearly reflects the financial problems of the past few years. For instance, the peak on June, 7, 2007 coincides with the announcement by Bear Stearns to the investors that it is suspending redemptions from its HighGrade Structured Credit Strategies Enhanced Leverage Fund. Two months later, in August 2007, the FIX peaks again. In this case, it goes along with with bankruptcy of American Home Mortgage Investment Corp. (NYSE: AHM) on August 6, 2007. The following days numerous quantitative long/short equity hedge funds suddenly began experiencing unprecedented losses. As such, on August 9, 2007, BNP Paribas SA, France's largest bank, suspended three investment funds because it could not "fairly" value their holdings after the U.S. subprime mortgage losses roiled credit markets. From 10 August 2007 on, the Central Banks around the world started injecting funds into markets as a response to an undesired and unwelcome spike in short-term rates. As a last example, we mention the huge increase in the Fear Index in October 2008 revealing the global financial crisis.

\subsection{Conclusions}

"Market fear" should be measured by several factors. In this research we have focused on three of them, which in our opinion, have a significant impact on the overall market fear level. First, we propose to take into account market risk and nervousness, expressed it in terms of the index volatility. The higher the volatility, the more market uncertainty there is and the wider the swings in the market can occur. Secondly, we propose to take into account the implied liquidity parameter intrinsically related to bid-ask spreads. Finally, we propose to measure the systemic risk and herd behavior via the comonotonicity ratio of the VIX and the VIX-ified comontonic upperbound. In a systemic crisis, all assets move into the same direction. The more comonotonic-like behavior we observe the more assets move together and the higher the systemic risk.

We presented the historical values of the market fear index solely based on vanilla index options and individual stock options. 


\section{References}

[1] Albrecher, H., Dhaene, J., Goovaerts, M. and Schoutens, W. (2005) Static Hedging of Asian Options under Lévy Models: The Comonotonicity Approach. Journal of Derivatives 12 (3), 63 - 72.

[2] Albrecher, H., Guillaume, F., Schoutens, W. (2011) Implied liquidity: model dependency investigation, Internal report.

[3] Black, F. and Scholes, M. (1973). The Pricing of Options and Corporate Liabilities. Journal of Political Economy 81 (3), 637-654.

[4] Britten-Jones, M. and Neuberger, A. (2000) Option prices, implied price processes and stochastic volatility. Journal of Finance 55, 839-866.

[5] Carr, P. and Madan, D. (1998), Towards a Theory of Volatility Trading, In: Volatility, Risk Publications, R. Jarrow, (edit.), 417-427

[6] Carr, P. and Wu,L. (2006) A tale of two indices. Journal of Derivatives 13 (3), 13-29.

[7] Chen, X., Deelstra, G., Dhaene, J., Vanmaele, M. (2008). Static Super-Replicating Strategies for a Class of Exotic Options. Insurance: Mathematics and Economics 42(3), 1067-1085.

[8] Cherny, A. and Madan, D. B. (2009). New Measures of Performance Evaluation, Review of Financial Studies 22, 2571-2606.

[9] Cherny, A. and Madan, D. B. (2010) Markets as a Counterparty: An Introduction to Conic Finance, International Journal of Theoretical and Applied Finance 13 (8), 1149-1177.

[10] Chicago Board Options Exchange, Inc. (2009). The CBOE Volatility Index - VIX. White paper.

[11] Chicago Board Options Exchange, Inc. (2009). The CBOE S\&P:500 Implied Correlation Index. White paper.

[12] Corcuera, J.M., Guillaume, F., Leoni, P., Schoutens, W. (2009) Implied Levy Volatility. Quantitaive Finance, 383-393.

[13] Corcuera, J.M., Guillaume, F., Madan, D.B. and Schoutens, W. (2010), Implied Liquidity - towards liquidity modeling and liquidity trading. Eurandom Report.

[14] Deelstra, G., Dhaene, J. and Vanmaele, M. (2010) An overview of comonotonicity and its applications in finance and insurance. In: Advanced Mathematical Methods for Finance, Oksendal, B. and Nunno, G. (editors), Springer, Germany (Heidelberg).

[15] Demeterfi, K., Derman, E., Kamal, M. and Zhou, J. (1999), More than You Ever Wanted to Know about Volatility Swaps, Goldman Sachs Quantitative Strategies Research Notes.

[16] Dhaene, J., Denuit, M., Goovaerts, M.J., Kaas R. and Vyncke D. (2002), The concept of comonotonicity in Actuarial science and finance: theory, Insurance: Mathematics 85 Economics, 3-33.

[17] Dhaene, J., Denuit, M., Goovaerts, M.J., Kaas, R. and Vyncke, D. (2002) The concept of comonotonicity in Actuarial science and finance: applications, Insurance: Mathematics \& Economics, 133161.

[18] Dhaene, J., Linders, D., Schoutens, W. and Vyncke, D. (2011) Measuring implied herd behavior in stock markets. K.U.Leuven, Faculty of Business and Economics research report.

[19] Hobson, D., Laurence, P. and Wang, T.H (2005), Static-arbitrage upper bounds for the prices of basket options. Quantitative Finance 5 (4), 329-342. 
[20] Jiang, G.J. and Tian, Y.S. (2005). Model-free implied volatility and its information content. Rev. Financ. Stud. 18 (4), 1305-1342.

[21] Laurence, P. (2007) Hedging and Pricing of Generalized Spread Options and the Market Implied Comonotonicity Gap, Presented at the Workshop and Mid-Term Conference on Advanced Mathematical Methods for Finance, Vienna University.

[22] Linders, D., Dhaene, J. and Schoutens, W. (2011). Some results on comonotonicity based upper bounds for index options. Working paper.

[23] Madan, D. B. and Schoutens, W. (2011) Conic Financial Markets and Corporate Finance. International Journal of Theoretical and Applied Finance, to appear.

[24] Neuberger, A. (1990), Volatility Trading, London Business School working paper.

[25] Simon, S., Goovaerts, M. and Dhaene, J. (2001) An easy computable upper bound for the price of an arithmetic Asian option. Insurance: Mathematics \& Economics 26 (2), 175-183.

[26] Zhou, H. and Chesnes, M. (2003). VIX Index Becomes Model Free and Based on S\&P:500. Papers and Memos from the Federal Reserve Board). 